PROCEEDINGS OF THE AMERICAN MATHEMATICAL SOCIETY

Volume 126, Number 4, April 1998, Pages 1115-1125

S 0002-9939(98)04240-3

\title{
FELL BUNDLES OVER GROUPOIDS
}

\author{
ALEX KUMJIAN
}

(Communicated by Palle E. T. Jorgensen)

\begin{abstract}
We study the $\mathrm{C}^{*}$-algebras associated to Fell bundles over groupoids and give a notion of equivalence for Fell bundles which guarantees that the associated $\mathrm{C}^{*}$-algebras are strongly Morita equivalent. As a corollary we show that any saturated Fell bundle is equivalent to a semi-direct product arising from the action of the groupoid on a $\mathrm{C}^{*}$-bundle.
\end{abstract}

A $\mathrm{C}^{*}$-algebraic bundle (see $[\mathrm{F} 2, \S 11]$ ) over a locally compact group may be thought of as a continuous version of a group grading in a $\mathrm{C}^{*}$-algebra; one may regard the associated $\mathrm{C}^{*}$-algebra as a fairly general sort of crossed product of the fiber algebra over the neutral element by the group (in [LPRS] it is shown that the $\mathrm{C}^{*}$-algebra is endowed with a coaction by the group). There is a natural extension of this definition to groupoids (see $[\mathrm{Yg}]$ ) which when specialized to trivial groupoids (i.e. topological spaces) yields the more usual notion of $\mathrm{C}^{*}$-algebra bundle. This object is referred to below as a Fell bundle.

Closely related notions have appeared in the literature: in recent work [Yn], Yamanouchi studies the analogous notion in the von Neumann algebra setting under the name integrable coaction. Fell bundles are reminiscent of the $\mathrm{C}^{*}$-categories discussed in [GLR]. They are also presaged in [Re2, Def. 5.3] (the object is used to construct a strong Morita equivalence bimodule).

Since each fiber of a saturated Fell bundle may be regarded as a strong Morita equivalence bimodule, the theory of Fell bundles provides a natural locus for proving theorems related to strong Morita equivalence of the kind which appear in [MRW] and $[\operatorname{Re} 2]$.

In $\S 1$ we fix notation and review some well-known facts concerning groupoids, Banach bundles, Hilbert modules, and equivalence bimodules. The notion of Fell bundle is defined in $\S 2$ and some examples are discussed, including the semi-direct product which results from a groupoid acting on a $\mathrm{C}^{*}$-algebra bundle fibered over the unit space. In $\S 3$ the associated $\mathrm{C}^{*}$-algebra is constructed in the case that the groupoid is r-discrete; the norm is defined by an analog of the left regular representation (the resulting $\mathrm{C}^{*}$-algebra may be regarded as the reduced $\mathrm{C}^{*}$-algebra associated to the Fell bundle). Finally, a Morita equivalence theorem of the sort discussed above is proved (Th. 4.2). This is used to show that there is an action of a groupoid on a $\mathrm{C}^{*}$-algebra bundle obtained from a saturated Fell bundle so that the $\mathrm{C}^{*}$-algebra associated to the Fell bundle is strongly Morita equivalent to that

Received by the editors September 23, 1996.

1991 Mathematics Subject Classification. Primary 46L55, 46L45; Secondary 46L05.

(C) 1997 by the author 
of the semi-direct product (Cor. 4.5). See [Kt, Th. 8], [PR, Cor. 3.7], [Q1, Th. 3.1], [Q2, Cor. 2.7], and [Yn, Th. 7.8] for related results.

\section{Preliminaries}

1.1. Given a groupoid $\Gamma$, let $\Gamma^{0}$ denote the unit space and $r, s: \Gamma \rightarrow \Gamma^{0}$ denote the range and source maps (respectively); let $\Gamma^{2}$ denote the collection of composable pairs; write the inverse map $\gamma \mapsto \gamma^{*}$. We tacitly assume that all groupoids under discussion are locally compact and Hausdorff, that the structure maps are continuous, and that they admit left Haar systems (see [Re1]). Let $\Delta$ denote the transitive equivalence relation with unit space $\Delta^{0}=\{0,1\}$; write $\Delta=\left\{0,1, \partial, \partial^{*}\right\}$ where $s(\partial)=0$ and $r(\partial)=1$. A groupoid $\Gamma$ is said to be trivial if $\Gamma=\Gamma^{0}$.

1.2. Given a Banach bundle, $p: E \rightarrow X$, let $C_{c}(E)$ denote the collection of compactly supported continuous sections of $E$ and $C_{0}(E)$ denote the collection of continuous sections of $E$ vanishing at $\infty$. Note that $C_{0}(E)$ may be regarded as the completion of $C_{c}(E)$ in the supremum norm. For $x \in X$, let $E_{x}$ denote the fiber over $x, p^{-1}(x)$. We shall tacitly assume that the total space, $E$, of any Banach bundle under consideration is second countable and that the base space, $X$, is locally compact and Hausdorff; it follows that the base space is second countable and that both the fiber, $E_{x}$, and the associated Banach space, $C_{0}(E)$, are separable (see [F2, Prop. 10.10]). By a result of Doady and dal Soglio-Hérault ( [F2, appendix]), if $X$ is locally compact, $E$ has enough continuous sections; thus for every $e \in E$ there is $f \in C_{c}(E)$ such that $f(p(e))=e$.

1.3. Let $A$ be a $\mathrm{C}^{*}$-algebra and $V$ be a right $A$-module; $V$ is said to be a (right) pre-Hilbert $A$-module if it is equipped with an $A$ valued inner product $\langle\cdot, \cdot\rangle$ which satisfies the following conditions:

i. $\langle u, \lambda v+w\rangle=\lambda\langle u, v\rangle+\langle u, w\rangle$,

ii. $\langle u, v a\rangle=\langle u, v\rangle a$,

iii. $\langle v, u\rangle=\langle u, v\rangle^{*}$,

iv. $\langle v, v\rangle \geq 0$ and $\langle v, v\rangle=0$ only if $v=0$,

for all $u, v, w \in V, \lambda \in \mathbf{C}$, and $a \in A$. Say that $V$ is a (right) Hilbert $A$-module if it is complete in the norm $\|v\|=\|\langle v, v\rangle\|^{1 / 2}$. There is an analogous definition for left Hilbert $A$-modules (the inner product in this case is linear in the first variable). Given a right Hilbert $A$-module $V$, there is a left Hilbert $A$-module $V^{*}$ with a conjugate linear isometric isomorphism from $V$ to $V^{*}$, written $v \mapsto v^{*}$, which is compatible with the module structure and inner product in the following way:

$$
\begin{gathered}
a v^{*}=\left(v a^{*}\right)^{*}, \\
\left\langle u^{*}, v^{*}\right\rangle=\langle u, v\rangle,
\end{gathered}
$$

for all $u, v \in V$ and $a \in A$. The term, Hilbert $A$-module, will be understood to mean right Hilbert $A$-module. If the span of the values of the inner product of a Hilbert $A$-module $V$ is dense in $A$, then $V$ is said to be full (see [Ks, $\S 2]$, [B, 13.1.1]). Note that $A$ may be regarded as a Hilbert $A$-module when endowed with the inner product $\langle a, b\rangle=a^{*} b$; evidently, $A$ is full. 
1.4. Given Hilbert $A$-modules $U$ and $V$, let $\mathcal{L}(V, U)$ denote the collection of bounded adjointable operators from $V$ to $U$ which commute with the right action of $A$. Put $\mathcal{L}(V)=\mathcal{L}(V, V)$; endowed with the operator norm, $\mathcal{L}(V)$ is a $\mathrm{C}^{*}$-algebra. For $u \in U, v \in V$, define $\theta_{u, v} \in \mathcal{L}(V, U)$ by $\theta_{u, v}(w)=u\langle v, w\rangle$ for $w \in V$. Note that $\theta_{u, v}=\theta_{v, u}^{*}$. The closure of the span of such operators is denoted $\mathcal{K}(V, U)$; write $\mathcal{K}(V)=\mathcal{K}(V, V)$. Note that $\mathcal{K}(V)$ is an (essential) ideal in $\mathcal{L}(V)$; in fact, $\mathcal{L}(V)$ may be identified with the multiplier algebra of $\mathcal{K}(V)$ (see [Ks, Th. 1]).

1.5. Given $\mathrm{C}^{*}$-algebras $A$ and $B$, a $B$ - $A$ equivalence bimodule $V$ is both a full right Hilbert $A$-module and a full left Hilbert $B$-module with compatible inner products ( $V^{*}$ may be viewed as an $A-B$ equivalence bimodule); note that equivalence bimodules are also known as imprimitivity bimodules (see [Ri1, Def. 6.10]). If such a bimodule exists the two $\mathrm{C}^{*}$-algebras are said to be strongly Morita equivalent (see [Ri2]). If $V$ is a full Hilbert $A$-module, then $V$ is a $\mathcal{K}(V)-A$ equivalence bimodule with $\mathcal{K}(V)$ valued inner product: $\langle u, v\rangle=\theta_{u, v}$. Indeed, every equivalence bimodule is of this form. Moreover, if $U$ and $V$ are full Hilbert $A$-modules then $\mathcal{K}(V, U)$ may be regarded as a $\mathcal{K}(U)-\mathcal{K}(V)$ equivalence bimodule.

1.6. Given $\mathrm{C}^{*}$-algebras $A, B$, and $C$ together with a $B$ - $A$ equivalence bimodule $V$ and a $C$ - $B$ equivalence bimodule $U$, one may form the $C$ - $A$ equivalence bimodule: $U \otimes_{B} V$ (see [Ri1, Th. 5.9]). Note that if $V$ is a $B$ - $A$ equivalence bimodule and $W$ is a $C$ - $A$ equivalence bimodule then one may identify

$$
W \otimes_{A} V^{*}=\mathcal{K}(V, W)
$$

(as $C$ - $B$ equivalence bimodules) via the map $w \otimes v^{*} \mapsto \theta_{u, v}$. By this identification and the associativity of the tensor product one obtains $\mathcal{K}\left(U \otimes_{B} V\right) \cong \mathcal{K}(U)$ :

$$
\begin{aligned}
\mathcal{K}\left(U \otimes_{B} V\right) & =\left(U \otimes_{B} V\right) \otimes_{A}\left(U \otimes_{B} V\right)^{*} \cong\left(U \otimes_{B}\left(V \otimes_{A} V^{*}\right)\right) \otimes_{B} U^{*} \\
& \cong\left(U \otimes_{B} B\right) \otimes_{B} U^{*} \cong U \otimes_{B} U^{*}=\mathcal{K}(U) .
\end{aligned}
$$

Note that this gives an isomorphism of $\mathrm{C}^{*}$-algebras; in fact, $K\left(U \otimes_{B} V\right) \cong C \cong$ $\mathcal{K}(U)$.

1.7. Given a $\mathrm{C}^{*}$-algebra bundle $A$ over a space $X$, a Banach bundle $V$ over $X$ is said to be a Hilbert $A$-module bundle if each fiber $V_{x}$ is a Hilbert $A_{x}$-module with continuous module action and inner product. Equipped with the natural inner product, $C_{0}(V)$ is a Hilbert $C_{0}(A)$-module, and it is full if and only if $V_{x}$ is full for every $x \in X$ (in this case $V$ is said to be full). Associated to $V$ one obtains a $\mathrm{C}^{*}$-algebra bundle $\mathcal{K}(V)$ where $\mathcal{K}(V)_{x}=\mathcal{K}\left(V_{x}\right)$. One has $C_{0}(\mathcal{K}(V)) \cong \mathcal{K}\left(C_{0}(V)\right.$ ).

\section{Fell Bundles}

We define below the natural analog of Fell's $\mathrm{C}^{*}$-algebraic bundles (cf. [F2], [Yg]) for groupoids. This notion is a generalization of both $\mathrm{C}^{*}$-algebraic bundles (over groups) and $\mathrm{C}^{*}$-algebra bundles (over spaces).

2.1. Let $\Gamma$ be a groupoid and $p: E \rightarrow \Gamma$ a Banach bundle; set

$$
E^{2}=\left\{\left(e_{1}, e_{2}\right) \in E \times E:\left(p\left(e_{1}\right), p\left(e_{2}\right)\right) \in \Gamma^{2}\right\} .
$$

Definition. A multiplication on $E$ is a continuous map, $E^{2} \rightarrow E$ (write $\left(e_{1}, e_{2}\right) \mapsto$ $\left.e_{1} e_{2}\right)$ which satisfies:

i. $p\left(e_{1} e_{2}\right)=p\left(e_{1}\right) p\left(e_{2}\right)$ for all $\left(e_{1}, e_{2}\right) \in E^{2}$,

ii. the induced map, $E_{\gamma_{1}} \times E_{\gamma_{2}} \rightarrow E_{\gamma_{1} \gamma_{2}}$, is bilinear for all $\left(\gamma_{1}, \gamma_{2}\right) \in \Gamma^{2}$, 
iii. $\left(e_{1} e_{2}\right) e_{3}=e_{1}\left(e_{2} e_{3}\right)$ whenever the multiplication is defined,

iv. $\left\|e_{1} e_{2}\right\| \leq\left\|e_{1}\right\|\left\|e_{2}\right\|$ for all $\left(e_{1}, e_{2}\right) \in E^{2}$.

An involution on $E$ is a continuous map, $E \rightarrow E$ (write $e \mapsto e^{*}$ ) which satisfies:

v. $p\left(e^{*}\right)=p(e)^{*}$ for all $e \in E$,

vi. the induced map, $E_{\gamma} \rightarrow E_{\gamma^{*}}$, is conjugate linear for all $\gamma \in \Gamma$,

vii. $e^{* *}=e$ for all $e \in E$.

Finally, the bundle $E$ together with the structure maps is said to be a Fell bundle if in addition the following conditions hold:

viii. $\left(e_{1} e_{2}\right)^{*}=e_{2}^{*} e_{1}^{*}$ for all $\left(e_{1}, e_{2}\right) \in E^{2}$,

ix. $\left\|e^{*} e\right\|=\|e\|^{2}$ for all $e \in E$,

x. $e^{*} e \geq 0$ for all $e \in E$.

Note that if $x \in \Gamma^{0}$ then $E_{x}$ is a $\mathrm{C}^{*}$-algebra (with norm, multiplication, and involution induced from the bundle); if $e \in E_{\gamma}$ then $e^{*} e \in E_{s(\gamma)}$, hence it makes sense to require that $e^{*} e$ be positive. Yamagami refers to such a bundle as a $\mathrm{C}^{*}$-algebra over a groupoid (see $[\mathrm{Yg}]$ ).

2.2. $\quad E$ is said to be nondegenerate if $E_{\gamma} \neq 0$ for all $\gamma \in \Gamma$; note that $E_{\gamma}$ is a right Hilbert $E_{s(\gamma)}$-module with inner product $\left\langle e_{1}, e_{2}\right\rangle=e_{1}^{*} e_{2}$ and a left Hilbert $E_{r(\gamma)}$-module with inner product $\left\langle e_{1}, e_{2}\right\rangle=e_{1} e_{2}^{*}$. Note also that $E_{\gamma}^{*} \cong E_{\gamma^{*}}$.

2.3. Given a Fell bundle $E$ over $\Gamma$, let $E^{0}$ denote the restriction $\left.E\right|_{\Gamma^{0}}$; clearly, $E^{0}$ is a $\mathrm{C}^{*}$-algebra bundle and $C_{0}\left(E^{0}\right)$ is a $\mathrm{C}^{*}$-algebra (with pointwise operations).

2.4. The Fell bundle $E$ is said to be saturated if $E_{\gamma_{1}} \cdot E_{\gamma_{2}}$ is total in $E_{\gamma_{1} \gamma_{2}}$ for all $\left(\gamma_{1}, \gamma_{2}\right) \in \Gamma^{2}$; note that if $E$ is saturated then $E_{\gamma}$ may be regarded as an $E_{r(\gamma)}-E_{s(\gamma)}$ equivalence bimodule (with inner products as above). For $\left(\gamma_{1}, \gamma_{2}\right) \in \Gamma^{2}$, one has $E_{\gamma_{1}} \otimes_{E_{x}} E_{\gamma_{2}} \cong E_{\gamma_{1} \gamma_{2}}$ where $x=s\left(\gamma_{1}\right)=r\left(\gamma_{2}\right)$ (via the map $e_{1} \otimes e_{2} \mapsto e_{1} e_{2}$ ).

\subsection{Examples.}

i. Let $E$ be a $C^{*}$-algebra bundle over a space $X$. If we regard $X$ as a trivial groupoid (so $X=X^{0}$ ), then $E$ is seen to satisfy the above definition.

ii. Let $E$ be a $\mathrm{C}^{*}$-algebraic bundle over a locally compact group $G$ (in the sense of Fell). If we regard $G$ as a groupoid (so $G^{0}=1_{G}$ ) then $E$ is easily seen to satisfy the above definition (it is essentially the same definition).

iii. Let $A$ and $B$ be $\mathrm{C}^{*}$-algebras and let $C$ be a $B$-A equivalence bimodule. Form a Fell bundle $E$ over the groupoid $\Delta$ as follows: set $E_{0}=A, E_{1}=B, E_{\partial}=C$, $E_{\partial^{*}}=C^{*}$; since $\Delta$ is discrete the Banach bundle structure is trivial. One defines multiplication and involution in the obvious way and checks that the above conditions are satisfied. Note that $E$ is saturated.

iv. Let $\Sigma$ be a proper $\mathbf{T}$-groupoid over $\Gamma$ (see [Ku, Def. 2.2]). Form the associated line bundle: $E=\Sigma *_{\mathbf{T}} \mathbf{C}=(\Sigma \times \mathbf{C}) / \mathbf{T}\left(\right.$ where $\left.t(\sigma, z)=\left(t s, t^{-1} z\right)\right)$. One defines multiplication and involution as follows:

$$
\begin{gathered}
\left(\sigma_{1}, z_{1}\right)\left(\sigma_{2}, z_{2}\right)=\left(\sigma_{1} \sigma_{2}, z_{1} z_{2}\right), \\
(\sigma, z)^{*}=\left(\sigma^{*}, \bar{z}\right) ;
\end{gathered}
$$

one must also check that both are well-defined (cf. [F2, §12]).

v. An action of $\Gamma$ on a $\mathrm{C}^{*}$-algebra bundle, $q: A \rightarrow \Gamma^{0}$, is a continuous map (see $[\mathrm{Re} 2],[\mathrm{M}]): \alpha: \Gamma * A \rightarrow A($ where $\Gamma * A=\{(\gamma, a) \in \Gamma \times A: s(\gamma)=q(a)\})$ (write $\alpha(\gamma, a)=\alpha_{\gamma}(a)$ ) which satisfies the following conditions: 
a. $q\left(\alpha_{\gamma}(a)\right)=r(\gamma)$ for all $\gamma \in \Gamma$ and $a \in A_{s(\gamma)}$,

b. $\alpha_{\gamma}: A_{s(\gamma)} \rightarrow A_{r(\gamma)}$ is a ${ }^{*}$-isomorphism for all $\gamma \in \Gamma$,

c. $\alpha_{x}(a)=a$ for all $x \in \Gamma^{0}$ and $a \in A_{x}$,

d. $\alpha_{\gamma_{1} \gamma_{2}}(a)=\alpha_{\gamma_{1}}\left(\alpha_{\gamma_{2}}(a)\right)$ for all $\left(\gamma_{1}, \gamma_{2}\right) \in \Gamma_{2}$ and $a \in A_{s\left(\gamma_{2}\right)}$.

Form the semi-direct product (cf. [F2, §12]) $\Gamma \times{ }_{\alpha} A=\Gamma * A$, with multiplication and involution given by the formulas

$$
\begin{gathered}
\left(\gamma_{1}, a_{1}\right)\left(\gamma_{2}, a_{2}\right)=\left(\gamma_{1} \gamma_{2}, \alpha_{\gamma_{2}^{*}}\left(a_{1}\right) a_{2}\right), \\
(\gamma, a)^{*}=\left(\gamma^{*}, \alpha_{\gamma}\left(a^{*}\right)\right) .
\end{gathered}
$$

Note that as a Banach bundle, $\Gamma \times{ }_{\alpha} A$ is the pull-back of $A$ by $s$. It is routine to check that with this norm and the above operations, the semi-direct product, $\Gamma \times{ }_{\alpha} A$, is a Fell bundle over $\Gamma$.

vi. Let $H$ be a Hilbert bundle over a locally compact space $X$ with an inner product $\langle\cdot, \cdot\rangle$ which is conjugate linear in the first variable. Let $\Gamma$ denote the transitive groupoid $X \times X$ (with obvious structure maps). There is a natural Fell bundle over $\Gamma$ associated to $H$. Set $E_{(x, y)}=\mathcal{K}\left(H_{y}, H_{x}\right)$. The topology of $E$ is prescribed by giving a linear space of norm continuous sections which is dense in every fiber (see $[\mathrm{F} 2,10.4]$ ); for each pair of continuous sections, $\xi, \eta$, of $H$ define a continuous section $\theta_{\xi, \eta}$ of $E$ by the formula

$$
\theta_{\xi, \eta}(x, y) \zeta=\xi(x)\langle\eta(y), \zeta\rangle
$$

for all $(x, y) \in \Gamma, \zeta \in H_{y}$. The span of such sections determines a bundle topology for $E$. Multiplication is given by composition, and involution by the usual adjoint.

2.6. Remark. Let $j: \Omega \rightarrow \Gamma$ be a continuous groupoid morphism and $p: E \rightarrow \Gamma$ be a Fell bundle. The pull-back of $E$ by $j, j^{*}(E)=\Omega * E=\{(\omega, e) \in \Omega \times E: j(\omega)=$ $p(e)\}$, may be regarded as a Fell bundle over $\Omega$ in the obvious way.

\section{Construction of the associated $\mathrm{C}^{*}$-Algebra}

3.1. Assume that $\Gamma$ is an $\mathrm{r}$-discrete groupoid (see [Re1]). Let $p: E \rightarrow \Gamma$ be a Fell bundle; we construct the analog of the reduced $\mathrm{C}^{*}$-algebra $C_{r}^{*}(E)$ as in $[\mathrm{Ku}, \S 2]$ (in $[\mathrm{Yg}]$ the full $\mathrm{C}^{*}$-algebra is constructed). Given $f, g \in C_{c}(E)$, define multiplication and involution by means of the formulas

$$
\begin{gathered}
f g(\gamma)=\sum_{\gamma=\alpha \beta} f(\alpha) g(\beta), \\
f^{*}(\gamma)=f\left(\gamma^{*}\right)^{*} .
\end{gathered}
$$

With these operations $C_{c}(E)$ forms a *-algebra. Let $P: C_{c}(E) \rightarrow C_{c}\left(E^{0}\right)$ be the restriction map. Define a $C_{c}\left(E^{0}\right)$-valued inner product on $C_{c}(E)$ by $\langle f, g\rangle=$ $P\left(f^{*} g\right)$.

3.2. Proposition. With this inner product, $C_{c}(E)$ is a pre-Hilbert $C_{0}\left(E^{0}\right)$-module.

Proof. We verify that $\langle f, f\rangle$ is positive as an element of the $\mathrm{C}^{*}$-algebra $C_{0}\left(E^{0}\right)$ for every $f \in C_{c}(E)$ :

$$
\langle f, f\rangle(x)=f^{*} f(x)=\sum_{x=\alpha \beta} f^{*}(\alpha) f(\beta)=\sum_{x=s(\gamma)} f(\gamma)^{*} f(\gamma) \geq 0
$$


for all $x \in \Gamma^{0}$, and $f \in C_{c}(E)$; the same computation shows that if $\langle f, f\rangle=0$ then $f=0$. The remaining properties are left for the reader to verify.

For $f \in C_{c}(E)$, put $\|f\|_{2}=\|\langle f, f\rangle\|^{1 / 2}$ and denote the completion of $C_{c}(E)$ in this norm by $L^{2}(E)$ (which is now a Hilbert $C_{0}\left(E^{0}\right)$-module).

3.3. We show that $L^{2}(E)$ is the Hilbert module associated to a bundle of Hilbert modules over $\Gamma^{0}$ (see 1.7). For each $x \in \Gamma^{0}$ consider the Hilbert $E_{x}$-module $V_{x}=$ $\bigoplus_{x=s(\gamma)} E_{\gamma}$, with inner product

$$
\left\langle\sum_{x=s(\gamma)} c_{\gamma}, \sum_{x=s(\gamma)} d_{\gamma}\right\rangle=\sum_{x=s(\gamma)} c_{\gamma}{ }^{*} d_{\gamma} .
$$

One may obtain a bundle topology on the union of the fibers by using elements of $C_{c}(E)$ to provide continuous sections in the obvious way (given $f \in C_{c}(E)$, one obtains the section $\left.x \mapsto \sum_{x=s(\gamma)} f(\gamma) \in V_{x}\right)$; let $V$ denote the bundle obtained in this way. Then $V$ is a Hilbert $E^{0}$-module bundle; one has $C_{0}(V) \cong L^{2}(E)$ as Hilbert $C_{0}\left(E^{0}\right)$-modules. Since $E^{0} \subset V$, as Hilbert $E^{0}$-module bundles, $V$ is full. If $E$ is saturated, then for all $\beta \in \Gamma$ one has

$$
V_{r(\beta)} \otimes_{E_{r(\beta)}} E_{\beta} \cong V_{s(\beta)}
$$

via the identification

$$
\left(\sum_{s(\gamma)=r(\beta)} c_{\gamma}\right) \otimes e \mapsto \sum_{s(\gamma)=r(\beta)} c_{\gamma} e
$$

where $e \in E_{\beta}, c_{\gamma} \in E_{\gamma}$, and

$$
\sum_{s(\gamma)=r(\beta)} c_{\gamma} \in \bigoplus_{s(\gamma)=r(\beta)} E_{\gamma}=V_{r(\beta)}
$$

Moreover, one has

$$
V_{x}^{*}=\bigoplus_{x=r(\gamma)} E_{\gamma}
$$

(via the involution map) and

$$
E_{\beta} \otimes_{E_{s(\beta)}} V_{s(\beta)}{ }^{*} \cong V_{r(\beta)}{ }^{*} .
$$

3.4. Left multiplication by an element in $C_{c}(E)$ is a bounded operator with respect to the norm, $\|\cdot\|_{2}$, and hence extends to the completion. One checks that

$$
\langle f g, h\rangle=\left\langle g, f^{*} h\right\rangle
$$

for all $f, g, h \in C_{c}(E)$; hence, left multiplication by an element in $C_{c}(E)$ is adjointable and one obtains a *-monomorphism

$$
C_{c}(E) \rightarrow \mathcal{L}\left(L^{2}(E)\right) \text {. }
$$

Let $C_{r}^{*}(E)$ denote the completion of $C_{c}(E)$ with respect to the operator norm; since $C_{r}^{*}(E)$ is a closed *-subalgebra of $\mathcal{L}\left(L^{2}(E)\right)$, it is a $\mathrm{C}^{*}$-algebra. Moreover, for each $x \in \Gamma^{0}$ one has a representation

$$
\pi_{x}: C_{r}^{*}(E) \rightarrow \mathcal{L}\left(V_{x}\right)
$$

so that for each $a \in C_{r}^{*}(E),\|a\|=\sup \left\|\pi_{x}(a)\right\|$. Note: Every bounded continous complex-valued function $g$ on $\Gamma^{0}$ may be identified with an element of the multiplier 
algebra, $M\left(C_{r}^{*}(E)\right)$, as follows: for $f \in C_{c}(E)$ put $g f(\gamma)=g(r(\gamma)) f(\gamma)$; one checks that this defines an element of $\mathcal{L}\left(L^{2}(E)\right)$ which centralizes $C_{r}^{*}(E)$.

3.5. Examples. i. If $E$ is a Fell bundle over a trivial groupoid $X\left(\right.$ so $X=X^{0}$ ), then $E$ is a $\mathrm{C}^{*}$-algebra bundle and $C_{r}^{*}(E)=C_{0}(E)$.

ii. Refer to example 2.5iii above; if $C$ is a $B$ - $A$ equivalence bimodule and $E$ is the associated Fell bundle over $\Delta$, then $C_{r}^{*}(E)$ is the linking algebra associated to $C$ (cf. [BGR, Th. 1.1]).

iii. Refer to example 2.5iv and assume that $\Gamma$ is a principal r-discrete groupoid. Then $C_{r}^{*}(E)$ has a diagonal subalgebra (see $[\mathrm{Ku}, \S 2]$ ) isomorphic to $C_{0}\left(G^{0}\right)$; the twist invariant for the diagonal pair $\left(C_{r}^{*}(E), C_{0}\left(G^{0}\right)\right)$ is the inverse of $[\Sigma]$.

iv. Let $\Gamma$ be a transitive equivalence relation on a countable set (with the discrete topology) and $E$ be a saturated Fell bundle over $\Gamma$. Let $V$ be the Hilbert $E^{0}$ module bundle over $G^{0}$ described above (3.3); for every $x \in \Gamma^{0}, V_{x}$ is full, so $V_{x}$ is a $\mathcal{K}\left(V_{x}\right)-E_{x}$ equivalence bimodule (see 1.5). Now, since $E$ is saturated, it follows that for any $\gamma \in \Gamma$ with $r(\gamma)=x$ and $s(\gamma)=y$, one has $V_{x} \otimes_{E_{x}} E_{\gamma} \cong V_{y}$ (see 3.3). This induces a ${ }^{*}$-isomorphism $\mathcal{K}\left(V_{y}\right) \cong \mathcal{K}\left(V_{x}\right)$ (see 1.6). Moreover, for each $x \in G^{0}, \pi$ induces a ${ }^{*}$-isomorphism:

$$
C_{r}^{*}(E) \cong \mathcal{K}\left(V_{x}\right) .
$$

3.6. Proposition. The restriction map, $P: C_{c}(E) \rightarrow C_{c}\left(E^{0}\right)$, extends to a conditional expectation $P: C_{r}^{*}(E) \rightarrow C_{0}\left(E^{0}\right)$.

Proof. This follows from Tomiyama's characterization of a conditional expection as a projection of norm one onto a subalgebra (see $[\mathrm{T}]$ ). One checks that, for $f \in C_{c}(E)$,

$$
\langle P(f), P(f)\rangle(x)=f(x)^{*} f(x) \leq \sum_{x=s(\gamma)} f(\gamma)^{*} f(\gamma)=\langle f, f\rangle(x)
$$

for each $x \in \Gamma^{0}$; hence, $\|P(f)\|^{2} \leq\|f\|^{2}$. Thus, $P$ extends to a projection $q \in$ $\mathcal{L}\left(L^{2}(E)\right)$; note that $q$ is a projection onto a Hilbert submodule isomorphic to $C_{0}\left(E^{0}\right)$. If $f \in C_{c}(E)$ is regarded as a left multiplication operator, then $\|P(f)\|=$ $\|q f q\| \leq\|f\|$. Hence, $P$ extends uniquely to a linear map, also denoted $P$, from $C_{r}^{*}(E)$ to $C_{0}\left(E^{0}\right)$ which restricts to the identity on $C_{0}\left(E^{0}\right)$, and $P$ is a projection of norm one.

3.7. Corollary. For all $f \in C_{c}(E),\|f\|_{2} \leq\|f\|$; thus, the inclusion, $C_{c}(E) \subset$ $L^{2}(E)$, extends to a continuous map,

$$
\iota: C_{r}^{*}(E) \rightarrow L^{2}(E)
$$

Proof. For all $f \in C_{c}(E),\left(\|f\|_{2}\right)^{2}=\left\|P\left(f^{*} f\right)\right\| \leq\left\|f^{*} f\right\|=\|f\|^{2}$.

3.8. Definition. An open subset $U \subset \Gamma$ is said to be an open $\Gamma$-set if the restrictions, $\left.r\right|_{U}$ and $\left.s\right|_{U}$, are one-to-one. An element $f \in C_{c}(E)$ is said to be a normalizer if supp $f$ is contained in an open $\Gamma$-set; let $\mathcal{N}(E)$ denote the collection of all normalizers. Note: Since $C_{c}(E)=\operatorname{span} \mathcal{N}(E), \mathcal{N}(E)$ is total in $C_{r}^{*}(E)$ and $L^{2}(E)$.

3.9. Fact. If $g \in C_{c}\left(E^{0}\right)$ and $f \in \mathcal{N}(E)$, then $f^{*} g f \in C_{c}\left(E^{0}\right)$. 
Proof. For each $x \in \Gamma^{0}$ there is at most one $\gamma \in \Gamma$ so that $s(\gamma)=x$ and $f(\gamma) \neq 0$. For $\beta \in \Gamma$ with $s(\beta)=x$, if $\beta=x$ and there is such a $\gamma$, one has

$$
f^{*} g f(\beta)=\sum_{\beta=\gamma_{1} \gamma_{2} \gamma_{3}} f^{*}\left(\gamma_{1}\right) g\left(\gamma_{2}\right) f\left(\gamma_{3}\right)=f(\gamma)^{*} g(r(\gamma)) f(\gamma) ;
$$

if $\beta \neq x$ or there is no such $\gamma$, each term in the above sum is zero and one has $f^{*} g f(\beta)=0$. Thus, $f^{*} g f \in C_{c}\left(E^{0}\right)$.

3.10. Proposition. $P: C_{r}^{*}(E) \rightarrow C_{0}\left(E^{0}\right)$ is faithful; thus, $\iota: C_{r}^{*}(E) \rightarrow L^{2}(E)$ is injective.

Proof. We will show that $P\left(a^{*} a\right) \neq 0$ for every $a \in C_{r}^{*}(E), a \neq 0$. For all $b \in C_{r}^{*}(E)$ and $f \in \mathcal{N}(E)$ we have

$$
f^{*} P(b) f=P\left(f^{*} b f\right)=\langle b f, f\rangle .
$$

It suffices to check this for $b \in C_{c}(E)$ where it follows by a calculation similar to that in the above fact. Since $\mathcal{N}(E)$ is total in $L^{2}(E)$, there is $f \in \mathcal{N}(E)$ so that $a f \neq 0$; by the above with $b=a^{*} a$,

$$
f^{*} P\left(a^{*} a\right) f=\left\langle a^{*} a f, f\right\rangle=\langle a f, a f\rangle \neq 0 .
$$

Hence $P\left(a^{*} a\right) \neq 0$ and $P$ is faithful.

3.11. Fact. The norm on $C_{c}(E)$ is the unique $\mathrm{C}^{*}$-norm extending the supremum norm on $C_{c}\left(E^{0}\right)$ for which $P$ extends to the completion as a faithful conditional expectation.

Proof. Let $A$ denote the completion of $C_{c}(E)$ in such a norm. $P$ extends to a conditional expectation, so left multiplication in $C_{c}(E)$ extends to a continuous *homomorphism, $A \rightarrow \mathcal{L}\left(L^{2}(E)\right)$; since $P$ is faithful the map must be injective.

3.12. Let $\Omega$ be an open subgroupoid of $\Gamma$; denote the inclusion map $j: \Omega \rightarrow \Gamma$ and put $D=j^{*}(E)$.

Proposition. The inclusion $C_{c}(D) \subset C_{c}(E)$ is isometric and thus extends to an inclusion $C_{r}^{*}(D) \subset C_{r}^{*}(E)$.

Proof. That the inclusion extends to a *homomorphism is immediate. The norm on $C_{c}(D)$ may be characterized as the $\mathrm{C}^{*}$-norm which extends the supremum norm on $C_{c}\left(D^{0}\right)$ for which $P$ is faithful on the completion. Since $P$ commutes with the inclusion $C_{c}(D) \subset C_{c}(E)$ and $P$ is faithful on the closure of $C_{c}(D)$ in $C_{r}^{*}(E)$, the inclusion is isometric.

3.13. Observe that $\|f\|_{\infty} \leq\|f\|_{2} \leq\|f\|$ for every $f \in C_{c}(E)$.

\section{Morita Equivalence}

We continue to restrict attention to principal r-discrete groupoids. Let $E$ be a saturated Fell bundle over a groupoid, $\Gamma$, and $V$ be the associated Hilbert $E^{0}$ module bundle over $\Gamma^{0}$ (see 3.3), we show below that there is an action $\sigma$ of $\Gamma$ on the $\mathrm{C}^{*}$-bundle $\mathcal{K}(V)$ so that $C_{r}^{*}\left(\Gamma \times{ }_{\sigma} \mathcal{K}(V)\right)$ and $C_{r}^{*}(E)$ are strongly Morita equivalent. 
4.1. Definition. A groupoid morphism, $\varphi: \Gamma \rightarrow \Delta$, is said to be full if for every $x \in \Gamma^{0}$ there is $\gamma \in \Gamma$ with $\varphi(\gamma) \notin \Delta^{0}$ such that $s(\gamma)=x$. For $i=0,1$ set $\Gamma_{i}=\varphi^{-1}(i)$ and note that $\Gamma_{i}$ is an open subgroupoid of $\Gamma$; note further that $\Gamma_{0}$ and $\Gamma_{1}$ are equivalent (see [MRW]). Let $j_{i}: \Gamma_{i} \rightarrow \Gamma$ denote the embeddings; if $E$ is a Fell bundle over $\Gamma$, put $E_{i}=j_{i}^{*}(E)$.

4.2. Theorem. Let $\Gamma$ be a groupoid, $\varphi: \Gamma \rightarrow \Delta$ be a full groupoid morphism, and $E$ a saturated Fell bundle over $\Gamma$. Then, with notation as above, $C_{r}^{*}\left(E_{0}\right)$ and $C_{r}^{*}\left(E_{1}\right)$ are strongly Morita equivalent (cf. [Re2, Cor. 5.4]).

Proof. By Prop. 3.12 we may regard $C_{r}^{*}\left(E_{0}\right)$ and $C_{r}^{*}\left(E_{1}\right)$ as subalgebras of $C_{r}^{*}(E)$. We show below that $C_{r}^{*}\left(E_{0}\right)$ and $C_{r}^{*}\left(E_{1}\right)$ are complementary full corners in $C_{r}^{*}(E)$; it will then follow (see [BGR, Th. 1.1]) that they are strongly Morita equivalent. It is clear that these subalgebras are complementary corners (as in 3.4 the characteristic functions on the unit spaces of $\Gamma_{0}$ and $\Gamma_{1}$ may be identified with complementary projections in $M\left(C_{r}^{*}(E)\right)$ ), and so it remains to show that they are full. By symmetry we need only show that $C_{r}^{*}\left(E_{0}\right)$ is contained in the ideal generated by $C_{r}^{*}\left(E_{1}\right)$. It suffices to show that $C_{c}\left(\left(E_{0}\right)^{0}\right)$ is contained in this ideal. Since $\varphi$ is full, for every $x \in\left(\Gamma_{0}\right)^{0}$ there is $\gamma \in \Gamma$ with $\varphi(\gamma)=\partial$ and $s(\gamma)=x$. Choose an open $\Gamma$-set $U$ containing $\gamma$ so that $U \subset \varphi^{-1}(\partial)$; every $g \in C_{c}(E)$ with supp $g \subset \varphi^{-1}(\partial)$ is in the ideal generated by $C_{r}^{*}\left(E_{1}\right)$, since supp $g g^{*} \subset \varphi^{-1}\left(\partial \partial^{*}\right)=\Gamma_{1}$. Since $E$ is saturated we may regard the restriction of $E$ to $U$ as a full Hilbert module bundle over the restriction of $\left(E_{0}\right)^{0}$ to $s(U)$. Hence, given $f \in C_{c}\left(\left(E_{0}\right)^{0}\right)$ with supp $f \in s(U)$ and $\epsilon>0$, there are $g_{k}, h_{k} \in C_{c}(E)$ with $\operatorname{supp} g_{k}, \operatorname{supp} h_{k} \subset U$ for $k=1, \ldots, n$ such that

$$
\left\|f-\sum_{1 \leq k \leq n} g_{k}^{*} h_{k}\right\|<\epsilon
$$

Hence $f$ is in the ideal generated by $C_{r}^{*}\left(E_{1}\right)$. Since each element in $C_{c}\left(\left(E_{0}\right)^{0}\right)$ can be written as the finite sum of such elements, $C_{c}\left(\left(E_{0}\right)^{0}\right)$ is contained in the ideal as desired.

4.3. Let $E$ be a saturated Fell bundle over $\Gamma$ and $V$ be the associated Hilbert $E_{0}$-module bundle over $\Gamma_{0}$; we construct another Fell bundle $F$ using $V$; for $\gamma \in \Gamma$ set

$$
F_{\gamma}=V_{r(\gamma)} \otimes_{E_{r(\gamma)}} E_{\gamma} \otimes_{E_{s(\gamma)}} V_{s(\gamma)}^{*}
$$

note that $F_{x}=V_{x} \otimes_{E_{x}} V_{x}^{*}=\mathcal{K}\left(V_{x}\right)$. Involution is defined in the obvious way:

$$
u \otimes e \otimes v^{*} \mapsto v \otimes e^{*} \otimes u^{*} ;
$$

given $(\alpha, \beta) \in \Gamma^{2}$, if $t \otimes d \otimes u^{*} \in F_{\alpha}$ and $v \otimes e \otimes w^{*} \in F_{\beta}$, define multiplication by the formula

$$
\left(t \otimes d \otimes u^{*}\right)\left(v \otimes e \otimes w^{*}\right)=t \otimes d\langle u, v\rangle e \otimes w^{*} \in F_{\alpha \beta} .
$$

The verification of the Fell bundle properties is straightforward (associativity follows from the associativity of the tensor product of equivalence bimodules - see [Ri1, Prop. 6.21]). Since $E$ is saturated and $V$ is full, $F$ is saturated. 
4.4. The following proposition is analogous to [Yn, Th. 5.3], in which the existence of an action, which is then defined to be the dual action of a given coaction (see [Yn, Def. 5.4]), is established.

Proposition. Let $F$ be as above. There is an action, $\sigma: \Gamma * \mathcal{K}(V) \rightarrow \mathcal{K}(V)$, so that $F \cong \Gamma \times{ }_{\sigma} \mathcal{K}(V)$.

Proof. First we identify $F$ with the pull-back bundle, $\Gamma * \mathcal{K}(V)$, by means of the following (see 3.3):

$$
F_{\gamma}=\left(V_{r(\gamma)} \otimes_{E_{r(\gamma)}} E_{\gamma}\right) \otimes_{E_{s(\gamma)}} V_{s(\gamma)}^{*} \cong V_{s(\gamma)} \otimes_{E_{s(\gamma)}} V_{s(\gamma)}^{*} \cong \mathcal{K}\left(V_{s(\gamma)}\right) ;
$$

the action $\sigma_{\gamma}: \mathcal{K}\left(V_{s(\gamma)}\right) \rightarrow \mathcal{K}\left(V_{s(\gamma)}\right)$ is likewise defined by the isomorphism (see also 3.5iv):

$$
\mathcal{K}\left(V_{s(\gamma)}\right) \cong F_{\gamma}=V_{r(\gamma)} \otimes_{E_{r(\gamma)}}\left(E_{\gamma} \otimes_{E_{s(\gamma)}} V_{s(\gamma)}^{*}\right) \cong V_{r(\gamma)} \otimes_{E_{r(\gamma)}} V_{r(\gamma)}^{*} \cong \mathcal{K}\left(V_{r(\gamma)}\right) .
$$

On elementary tensors of the form $a c \otimes b^{*} \in \mathcal{K}\left(V_{s(\gamma)}\right)$, where $a \in E_{\alpha}, b \in E_{\beta}, c \in E_{\gamma}$, and $s(\alpha)=r(\gamma), s(\beta)=s(\gamma)$ (note that $a c \in E_{\alpha \gamma} \subset V_{s(\gamma)}$ and $b \in E_{\beta} \subset V_{s(\gamma)}$, and that elements of the form, $a c \otimes b^{*}$ span a dense subset of $\left.\mathcal{K}\left(V_{s(\gamma)}\right)\right), \sigma_{\gamma}$ is given by

$$
\sigma_{\gamma}\left(a c \otimes b^{*}\right)=a \otimes c b^{*} .
$$

It is a routine matter to verify that $\sigma$ defines an action of $\Gamma$ on $\mathcal{K}(V)$ (for example, $\sigma_{x}=\operatorname{id}_{\mathcal{K}\left(V_{x}\right)}$ for $x \in \Gamma^{0}$ follows from the fact that $\mathcal{K}\left(V_{x}\right)=V_{x} \otimes_{E_{x}} V_{x}^{*}$ is a balanced tensor product) and that $F \cong \Gamma \times_{\sigma} \mathcal{K}(V)$.

4.5. Corollary. With notation as above, $C_{r}^{*}(E)$ and $C_{r}^{*}\left(\Gamma \times{ }_{\sigma} \mathcal{K}(V)\right)$ are strongly Morita equivalent.

Proof. To apply Th. 4.2, we construct a Fell bundle $D$ over $\Gamma \times \Delta$ which restricts to $E$ on $\Gamma \times 0$ and to $F$ on $\Gamma \times 1$ (by Prop. 4.4, $F \cong \Gamma \times_{\sigma} \mathcal{K}(V)$ ). For $\gamma \in \Gamma$, define $D_{(\gamma, 0)}=E_{\gamma}, D_{(\gamma, 1)}=F_{\gamma}, D_{(\gamma, \partial)}=V_{r(\gamma)} \otimes_{E_{r(\gamma)}} E_{\gamma}$, and $D_{\left(\gamma, \partial^{*}\right)}=E_{\gamma} \otimes_{E_{s(\gamma)}}$ $V_{s(\gamma)}^{*}$. One defines multiplication and involution in the natural way. For example, if $(\alpha, \beta) \in \Gamma^{2}$ the map

$$
D_{\left(\alpha, \partial^{*}\right)} \times D_{(\beta, \partial)} \rightarrow D_{(\alpha \beta, 0)}=E_{\alpha \beta}
$$

is given by the formula

$$
\left(d \otimes u^{*}\right)(v \otimes e)=d\langle u, v\rangle e
$$

or the map

$$
D_{(\alpha, \partial)} \times D_{\left(\beta, \partial^{*}\right)} \rightarrow D_{(\alpha \beta, 1)}=F_{\alpha \beta}=V_{r(\alpha)} \otimes_{E_{r(\alpha)}} E_{\alpha \beta} \otimes_{E_{s(\beta)}} V_{s(\beta)}^{*},
$$

is given by the formula

$$
(v \otimes e)\left(d \otimes u^{*}\right)=v \otimes e d \otimes u^{*} .
$$

Note that $D$ is saturated. Thus, the theorem applies (the map, $\Gamma \times \Delta \rightarrow \Delta$, is given by projection onto the second factor).

4.6. Remark. If the groupoid is a (discrete) group this result may be obtained by combining [Q2, Cor. 2.7] and [Kt, Th. 8]; I wish to thank Quigg for bringing this to my attention. 


\section{REFERENCES}

[B] B. Blackadar, K-theory for operator algebras, MSRI Publication Series 5, Springer-Verlag (1986). MR 88g:46082

[BGR] L. G. Brown, P. Green and M. A. Rieffel, Stable isomorphism and strong Morita Equivalence of $C^{*}$-algebras, Pacific J. Math. 21 (1977) 349-363. MR 57:3866

[GLR] P. Ghez, R. Lima and J. E. Roberts, $W^{*}$-categories, Pacific J. Math. 120 (1985) 79-109. MR 87g:46091

[F1] J. M. G. Fell, An extension of Mackey's method to Banach *-algebraic bundles, Mem. Amer. Math. Soc. 90 (1969). MR 41:4255

[F2] J. M. G. Fell, Induced representations and Banach *-algebraic bundles, Springer Lecture Notes in Mathematics 582 (1977). MR 56:15825

[FD] J. M. G. Fell and R. S. Doran, Representations of *-algebras, locally compact groups, and Banach *-algebraic bundles, Vol. 2, Academic Press (1988). MR 90c:46002

[Ks] G. G. Kasparov, Hilbert $C^{*}$-modules: theorems of Stinespring and Voiculescu, J. Operator Theory 4 (1980) 133-150. MR 82b:46074

[Kt] Y. Katayama, Takesaki's duality for a nondegenerate co-action, Math. Scand. 55 (1984) 141-151. MR 86b:46112

[Ku] A. Kumjian, On $C^{*}$-diagonals, Can. J. Math. 38 (1986) 969-1008. MR 88a:46060

[LPRS] M. B. Landstad, J. Phillips, I. Raeburn and C. E. Sutherland, Representations of crossed products by coactions and principal bundles, Trans. Amer. Math. Soc. 299 (1987) 747784. MR 88f: 46127

[M] T. Masuda, Groupoid dynamical systems and crossed product. II: the case of $C^{*}$-systems, Publ. Res. Inst. Math. Sci.20 (1984) 959-970. MR 86g:46100

[MRW] P. S. Muhly, J. N. Renault and D. P. Williams, Equivalence and isomorphism for groupoid $C^{*}$-algebras, J. Operator Theory 17 (1987) 3-22. MR 88h:46123

[PR] J. A. Packer and I. Raeburn, Twisted crossed products of $C^{*}$-algebras, Math. Proc. Cambridge Philos. Soc. 106 (1989) 293-311. MR 90g:46097

[Q1] J. C. Quigg, Duality for reduced twisted crossed products of $C^{*}$-algebras, Indiana Univ. Math. J. 35 (1986) 549-571. MR 88d:46122

[Q2] J. C. Quigg, Discrete $C^{*}$-coactions and $C^{*}$-algebraic bundles, J. Austral. Math. Soc. (Ser. A) 60 (1996), 204-221. MR 97c:46086

[Re1] J. Renault, A groupoid approach to $C^{*}$-algebras, Springer Lecture Notes in Mathematics 793 (1980). MR 82h:46075

[Re2] J. Renault, Représentations des produits croisés d'algèbres de gropoïdes, J. Operator Theory 18 (1987) 67-97. MR 89g:46108

[Ri1] M. A. Rieffel, Induced Representations of $C^{*}$-algebras, Advances Math. 13 (1974) 176257. MR 50:5489

[Ri2] M. A. Rieffel, Morita equivalence for operator algebras, Operator algebras and applications (R. Kadison, ed.), Proc. Symp. Pure Math. Vol. 38, Part 1, Amer. Math. Soc., 1982) 285-298. MR 84k:46045

[T] J. Tomiyama, On the projection of norm one in $W^{*}$-algebras, Proc. Japan Acad. 33 (1957) 608-612. MR 20:2635

[Yg] S. Yamagami, On primitive ideal spaces of $C^{*}$-algebras over certain locally compact groupoids, Mappings of operator algebras (H. Araki and R. Kadison, eds.), Progress in Math. Vol. 84, Birkhäuser, Boston, (1991) 199-204. MR 92j:46110

[Yn] T. Yamanouchi, Duality for actions and coactions of measured groupoids on von Neumann Algebras, Mem. Amer. Math. Soc. 484 (1993). MR 94b:46095

Department of Mathematics, University of Nevada, Reno, Nevada 89557

E-mail address: alex@unr.edu 